\title{
EFECTOS DEL VACIADO DEL EMBALSE DE JOAQUÍN COSTA SOBRE ANFIBIOS, AVES Y MAMÍFEROS VINCULADOS AL AMBIENTE DE RIBERA
}

\author{
C. Rosell ${ }^{1,2}$, J. Nadal ${ }^{1}$, S. Cahill ${ }^{2}$, R. Campeny ${ }^{1,2}$, J. Parpal $^{2}$, V. Pedrocchi ${ }^{1}$ y J. Ruíz-Olmo'. \\ 1. Dept. Biologia Animal (Vertebrats). Fac. Biologia. Univ de Barcelona. Av. Diagonal, 645. 08028 Barcelona. \\ 2. MINUARTIA, Estudis Ambientals. Pg. Domènech, 3, 1. 08470 Sant Celoni
}

\begin{abstract}
RESUMEN
El embalse de Joaquín Costa se sitúa en una zona de notable interés para la conservación de la biodiversidad. ya que contiene elementos faunísticos relevantes entre los que destacin la comunidad de aves acuáticas y la población de nutria (Lutra lutra).

La monitorización de los cambios derivados del efecto de los vaciados sobre las poblaciones del embalse y de los cursos del Ésera y del Cinca aguas abajo de la presa, se ha diseííado con el objetivo de mejorar el conocimiento de la respuesta de las comunidades faunísticas a esta perturbación y definir medidas para minimizar el impacto del vaciado de otros embalses. El aeguiniiento se ha centrado en los táxones más sensibles, que tienen mayor capacidad bioiiidicadora, concretamente anfibios. aves acuáticas y nutria.

Las aves acuáticas y la nutria muestran intensos cambios en el uso del espacio en las semanas posteriores al vaciado, mientras que en las prospecciones realizadas seis meses después, se aprecia una notable recuperación de la actividad en la mayor parte de las zonas afectadas (con la excepción de algunos tramos que siguen manteniendo un bajo índice de uso por parte de la nutria) y las comunidades de aves acuáticas muestran una composición similar a la de los muestreos previos al primer vaciado.

Se consideran factores clave en la minimización de los efectos del vaciado la época en la que se ha llevado a cabo (no afecta los períodos reproduetores principales de aves y de anfibios), la disponibilidad de hábitats alternativos accesibles durante el periodo de máxima alteración y la corta duración de la perturbación, favorecida por las abundantes precipitaciones posteriores a los vaciados, que comportaron un efecto de arrastre de sedimentos y limpieza del lecho fluvial. Para posteriores vaciado?, se recomienda la elaboración de un estudio previo detallado, ya que el diseño de medidas preventivas y correctoras debe realizarse de manera particularizada para cada caso.
\end{abstract}

Palabras clave: vaciado de un embalse, impacto ambiental, Lutra lutra, vertebradoa acuáticos.

\section{SUMMARY}

Joaquin Costa reservoir is situated in an area of great interest for biodiversity conservation, given that it contains an outstanding fauna among which the aquatic bird community and the otter population (Lutra lutra) stand out.

The monitoring of the changes brought about by the effects of the dam drainages on the populations of the reservoir and the stretches of the Esera and the Cinca rivers downstream from the dam, was designed with the objective of improving the knowledge of the response of the faunal communities to this disturbance and to define measures to minimize the impact of the drainage of other reservoirs. Monitoring was centered on the most sensitive taxa which have the greatest bioindicative capacity, specifically amphibians, aquatic birds and the otter.

The aquatic birds and the otter showed intense changes in their use of space during the weeks following the flushing, whilst during the surveys undertaken six months afterwards, a considerable recovery was observed in the activity of most of the affected areas (with the exception of some stretches which continued to register a low index of use by the otter). The communitics of aquatic birds showed a composition which was similar to that of the surveys undertaken prior to the first flushing.

The following are considered as key factors in minimizing the effects of the flushing: the period during which the flushing were carried out (did not affect the principal reproductive period of birds and amphibians), the availability of accessible alternative habitats during the period of maximum alteration, and the short duration of the perturbation, which was favoured by the abundant rains following the drainages, as they washed away the sediments and cleaned the riverbed. For future flushing, it is recommended that a detailed study be elaborated previous to the event, given that the design of preventive and corrective measures needs to be undertaken in a specific manner for each case.

Key words: reservoir flushing, environmental impact, Lutra lutra, aquatic vertebrates 


\section{INTRODUCCIÓN}

Los ambientes de ribera constituyen enclaves de gran interés para la conservación de la biodiversidad y albergan una notable comunidad de vertebrados. La zona de estudio destaca en este sentido por el gran número de especies de aves acuáticas, y particularmente, por la población de nutria.

En la bibliografía especializada no se ha documentado ningún caso de estudio en el que se haya realizado un seguimiento de los efectos del vaciado de un embalse sobre las comunidades de los vertebrados de ribera o semi-acuáticos. Por ello, la monitorización de los cambios que se producen con posterioridad al vaciado del embalse de Joaquín Costa -también llamado de Barasona-, constituye una experiencia pionera, de la que se han podido extraer conclusiones de interés general para la prevención de los impactos de otras actuaciones similares. Los objetivos básicos del estudio de seguimiento realizado son concretamente los siguientes:

- Caracterizar el estado inicial (previo al vaciado) de las comunidades de vertebrados vinculados al ambiente de ribera y de las zonas de mayor interés faunístico.

- Evaluar los efectos del vaciado sobre los grupos taxonómicos estudiados.

- Identificar los aspectos que tienen una mayor incidencia en la prevención y corrección de impactos y plantear recomendaciones generales que puedan ser aplicables a otras actuaciones similares.

En la comunicación se resumen los principales resultados referentes a tres táxones especialmente destacados (anfibios, aves acuáticas y nutria), sin detallar el conjunto de los trabajos realizados.

\section{METODOLOGÍA Y ÁREA DE ESTUDIO}

\section{Zona de estudio}

El área de estudio básica comprende el embalse de Barasona, el curso del Ésera aguas abajo de la presa y el tramo del Cinca desde la confluencia hasta el azud Arias II. En el caso de la nutria, la zona de estudio se ha ampliado incorporando el tramo del Cinca entre el embalse del Grado y la confluencia,, y también se han prospectado un tramo del Esera aguas arriba del embalse de
Barasona, y del Cinca, aguas abajo del azud de Arias II.

\section{Grupos taxonómicos estudiados}

Ante la dificultad de realizar un análisis exhaustivo de todos los táxones de vertebrados presentes en el área de estudio, se han seleccionado los elementos faunísticos más sensibles o los que tienen mayor capacidad bioindicadora. En concreto se han monitorizado los efectos sobre los anfibios, las aves acuáticas y los mamíferos, con especial atención al caso de la nutria (Lutra lutra) (Fig. 34 del anexo), por tratarse de una especie semi-acuática amenazada y que es objeto de una fuerte protección a nivel legal.

\section{Métodos aplicados}

La mayor parte del estudio se ha llevado a cabo durante dos años: los trabajos se iniciaron en la primavera de 1994 y han finalizado en verano de 1996, excepto para el caso de la nutria en el que se ha realizado también un seguimiento durante la primavera de 1997, con posterioridad al segundo vaciado.

La metodología aplicada en cada uno de los grupos taxonómicos es distinta, debido a la necesidad de adaptarla a las peculiaridades eco-etológicas de cada grupo.

En el caso de los anfibios se establecieron siete recorridos por los márgenes de los cursos fluviales, de longitud variable entre 300 y $700 \mathrm{~m}$. Estos transectos se prospectaban durante la primavera inspeccionando los puntos adecuados para la reproducción o refugio, y anotando todas las especies observadas así como los aspectos fenológicos.

En el caso de las aves la metodología básica combina los censos en tres áreas discretas (embalse de Barasona y azudes de Arias I y Arias 11) y los censos en seis tramos fluviales de unos $500 \mathrm{~m}$ de longitud. Los censos se repitieron estacionalmente y también se desarrollaron prospecciones específicas en los períodos inmediatamente anterior y posterior al vaciado, con un total de 11 muestreos distribuidos entre el invierno de 1994 y la primavera de 1996.

El seguimiento de los efectos del vaciado sobre la población de nutria presenta mayor complejidad. La dificultad de observación directa de este mustélido, de hábitos crepusculares y nocturnos, ha requerido la aplicación de diversas téc- 
nicas. Descartada la posibilidad de aplicar radioseguimiento por las dificultades en la obtención de los permisos necesarios, se combinaron los siguientes métodos:

- Prospecciones generales que incluían la totalidad del área de estudio, con una media de $40 \mathrm{~km}$ prospectados en cada campaña combinando diversas técnicas basadas en la detección de rastros (Fig. 33 del anexo), ampliamente utilizadas en los estudios sobre la especie en toda su área de distribución (Mason \& Macdonald, 1986; Delibes, 1990; Sidorovich \& Lauzhel, 1992; Sidorovich, 1994). En ellas se registraban los indicios de actividad (huellas, excrementos y gelatinas, puntos de marcaje, madrigueras y encames) en distintos sectores. La información se completó con la aplicación de técnicas fotográficas y de filmaciones de vídeo con infrarrojos que permitieron determinar aspectos como el tamaño de una camada (Rosell et al., 1995; Jové, 1996). Las prospecciones se repitieron un mes antes y un mes después del vaciado, y posteriormente en la primavera de 1996 y en la de 1997.

- Prospecciones en estaciones concretas. En cuatro tramos fluviales se realizaron transectos de 400 a $600 \mathrm{~m}$ de longitud en los que se anotaban los rastros observados, siguiendo la metodología normalizada que se aplica en diversos países europeos (Lenton et al., 1980; Ruíz-Olmo, 1985; Delibes et al., 1991). Estos transectos se recorrían con periodicidad estaciona], siguiendo las prospecciones iniciadas en 1985, con independencia del presente estudio (Ruíz-Olmo, com pers.).

- Análisis de la dieta. Realizado a partir del análisis de los restos que se identificaban en los excrementos recogidos durante los recorridos siguiendo el método descrito por Ruíz-Olmo, J. (1995).

\section{RESULTADOS Y DISCUSIÓN}

\section{Anfibios}

En la zona de estudio se ha detectado la presencia de cinco especies de anfibios: el sapo partero (Alytes obstetricans), el sapo común (Bufo bufo) (Fig. $31 \mathrm{del}$ anexo), el sapo corredor (Bufo calamita), la ranita de San Antonio (Hyla arborea) y la rana verde común (Ranaperezi). Se trata de las mismas especies que han sido citadas hasta el momento en áreas cercanas a la zona de estudio (Falcón, 1982 y 1987, Martínez Rica, 1989). El período de reproducción y desarrollo larvario de estas especies se prolonga en la zona, desde finales de febrero hasta agosto, aunque los renacuajos de sapo partero y rana verde pueden permanecer en el agua durante más tiempo. Los sectores que muestran mayor diversidad de anfibios son el tramo final del curso del Esera, próximo a la Confluencia, y el Cinca, en el sector próximo a Estada. La mayor presencia de especies y de individuos se relaciona con la existencia de zonas de aguas lentas o estancadas situadas en el margen del curso principal y con presencia de vegetación helofítica y de ribera.

No se han detectado diferencias importantes en el número y localización de las especies detectadas en las tres campañas de prospección, aunque los resultados no son directamente comparables entre los tres períodos debido a que las fuertes riadas de los meses posteriores al primer vaciado provocaron fuertes cambios en los tramos de río prospectados y dificultades en el muestreo de anfibios. Cabe destacar no obstante, que los efectos sobre las poblaciones de anfibios se han visto atenuados por la época en que se produjo el vaciado, ya que el periodo reproductor había finalizado, y por el mantenimiento de extensas zonas de vegetación de ribera que constituyeron hábitats adecuados en la primavera posterior al vaciado.

Uno de los cambios más notables se produjo en la zona de la confluencia del Ésera y del Cinca que en el periodo posterior al vaciado mostró menor diversidad de especies reproductoras. Éste resultado se atribuye a la alteración de hábitats de esta zona, con pérdida de formaciones vegetales de ribera y de ambientes constituidos por pequeñas charcas marginales que fueron sustituidas por elementos como al azud y canal de derivación asociado a los caladeros. Aunque estas balsas constituyeron nuevos puntos de reproducción, su estructura sólo permite la presencia de puestas y renacuajos de sapo común. Se sustituyó por tanto, un ambiente heterogéneo que permitía la reproducción de diversas especies, por otro más homogéneo, dónde sólo se localizó una especie.

\section{Aves acuáticas}

La zona de estudio se caracteriza por una notable diversidad de especies de aves tal como indican los diversos estudios realizados hasta el 


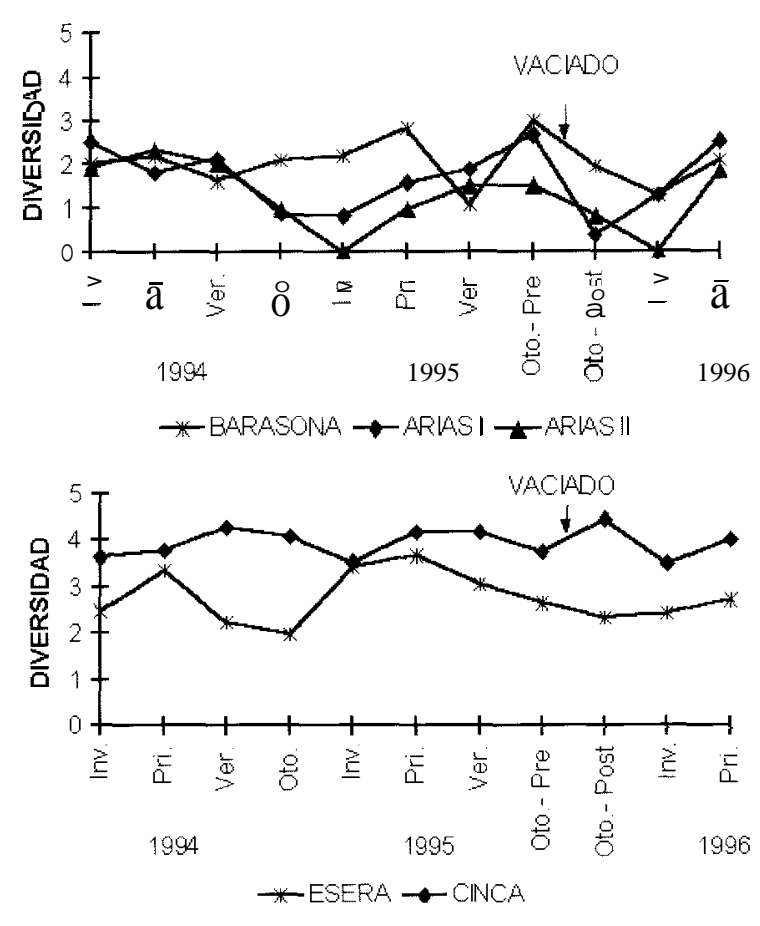

Figura 1. Valores del índice de diversidad de Shannon para las aves, obtenidos a partir de los censos efectuados en el embalse de Barasona, en Arias I, en Arias II y en los tramos fluviales de los ríos Ésera y Cinca, a lo largo del període estudiado. Se separan los censos de otoño de 1995 anterior y posterior al vaciado del embalse. Values of the Shannon diversity indexfor birds obtained from the censuses carried out throughout the study period at the Barasona reservoir, at Arias I, at Arias $I I$ and at the fluvial stretches of the Esera and Cinca rivers. The censuses of autumn 1995 prior to and following druinage of the reservoir are given separately.

momento en el embalse de Barasona (Bueno, 1993, Pedrocchi, 1978, SEO-Aragón, 1994). En los muestreos anteriores al vaciado se identificaron un total de 124 especies, destacando la riqueza de especies acuáticas (aproximadamente el $25 \%$ del total) y de rapaces diurnas.

La mayor riqueza de aves acuáticas se observa en el embalse de Barasona (20 especies detectadas en el período de estudio) y en el sector embalsado por el azud Arias 1 (21 especies).

El embalse es un enclave importante para la ornitofauna, siendo las especies más abundantes el cormorán grande (Phalacrocorax carbo), el ánade real (Anas platyrhynchos) (Fig. $32 \mathrm{del}$ anexo), la gaviota reidora (Larus ridibundus) y la gaviota patiamarilla (Larus cachinnans), acom- pañadas de limícolas, ardéidos, otras anátidas, e incluso especies tan singulares como el águila pescadora (Pandion haliaetus). La zona embalsada por el azud Arias I también presenta una comunidad de aves acuáticas relevante, destacando como punto de reproducción de somormujo lavanco (Podiceps cristatus) (Fig. $30 \mathrm{del}$ anexo) y de aguilucho lagunero (Circus aeruginosus).

Los dos cursos fluviales estudiados presentan características muy distintas; mientras que en el Esera predominan las especies de carácter rupícola, en el Cinca existe una comunidad de aves acuáticas muy diversificada, a la que se añaden especies propias de los espacios abiertos que bordean el cauce.

A nivel global, los efectos del vaciado fueron muy evidentes a corto plazo, observándose importantes variaciones en el número de individuos de aves acuáticas y en su distribución. Las especies más sensibles abandonaron las áreas afectadas, mientras que las oportunistas que se alimentaban de peces muertos o moribundos, incrementaron notablemente su presencia. En la primavera de 1996, las condiciones ambientales del embalse y de los cursos fluviales muestran una considerable recuperación, y la comunidad de aves acuáticas es similar a la de años anteriores, manteniéndose una diversidad que se encuentra dentro del rango de variación observado en los muestreos previos al vaciado (Fig. 1).

Cabe destacar que entre las prospecciones previas al vaciado se observaron variaciones importantes de densidad y número de especies (Figs. 2 y 3). Por este motivo, y en este contexto fluctuante, no se detectan variaciones especialmente significativas a medio plazo que puedan ser atribuibles al efecto del desembalse. No obstante, si se observaron importantes efectos inmediatos en compración con los que se detectaron a medio plazo.

- A corto plazo (un mes después del vaciado), las diferencias en la comunidad de aves son significativas tanto en el embalse como en Arias 1 y en el tramo fluvial del Cinca.

1. En el embalse de Barasona, los cormoranes invernantes y los somormujos lavancos no aparecen en el censo posterior al vaciado; estas aves requieren aguas profundas y limpias, con peces y por ello, con el embalse vacío se vieron obligadas a desplazarse a otros lugares. Se observó en cambio, un incremento de garzas reales (Ardea cine- 
BARASONA

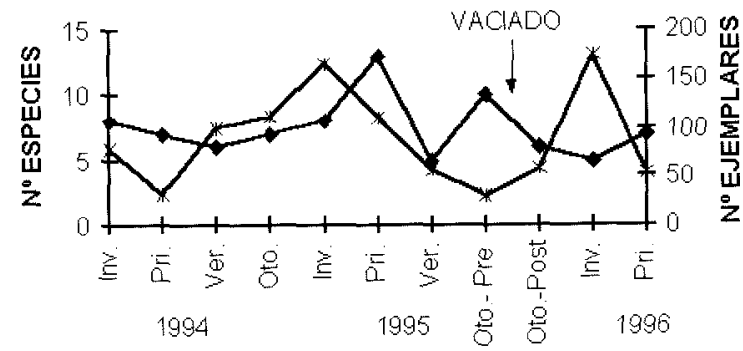

ARIAS I

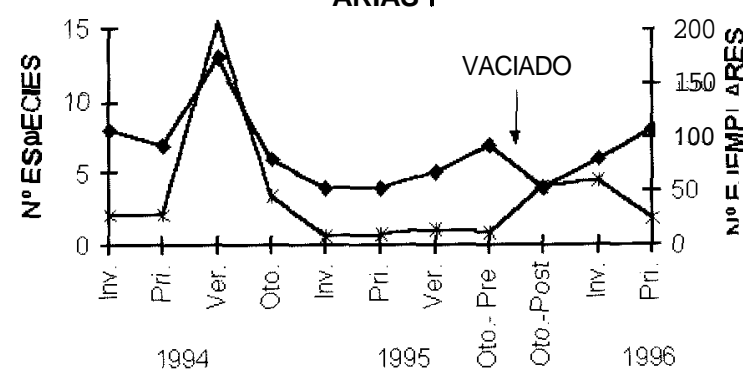

ARIAS II

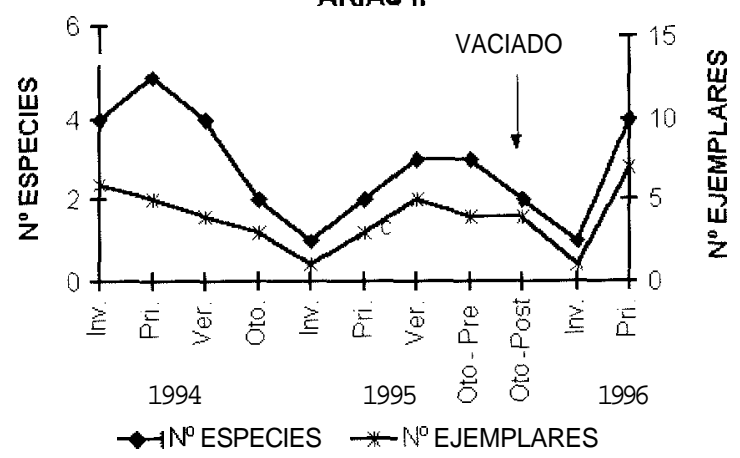

Figura 2. Número de especies de aves y número de ejemplares registrados en las diferentes estaciones de muestreo. Se separan los censos de otoño de 1995 anterior y posterior al vaciado del embalse. Nutnher of species of birds and the number of individuals registe\& at the different survey sites. The censuses of autumn 1995 prior tu and following drainuge of the reservoir are given separately.

rea) y de gaviotas patiamarillas, que aprovecharon el nuevo recurso trófico que constituían los peces que quedaron aislados en pequeñas charcas. Los efectivos poblacionales de ánades reales y gaviotas reidoras (ambas especies fácilmente adaptables a modificaciones del medio), se mantuvieron similares a censos anteriores, aunque con valores ligeramente inferiores.
2. En la zona embalsada por el azud Arias I, que en otoño de 1994 se encontraba llena de barro y sin agua, la comunidad de aves acuáticas evidenció un fuerte descenso de la diversidad, pasando a estar claramente dominada por la gaviota reidora. No se observaron ni zampullines ni ánades reales como en muestreos anteriores al vaciado.

3. En el río Cinca, en los primeros días posteriores al vaciado, aparecieron numerosas gaviotas patiamarillas, aves oportunistas que consumían los peces muertos, y las garzas reales, también de presencia generalizada en el cauce, que capturaban los peces debilitados y moribundos.

- A medio plazo (comparación inviernos y primaveras previos y posteriores al desembalse), no se manifiestan variaciones claramente atribuibles al efecto del vaciado.

1. En el embalse, la comunidad de aves invernantes muestra una situación similar a la de años anteriores. Los cormoranes vuelven a estar presentes, aunque con efectivos menos numerosos que en años anteriores, y las gaviotas reidoras mantienen su tendencia a aumentar, mientras que el ánade real muestra efectivos poblacionales similares a los que tenía en el periodo prevaciado.

2. En el azud Arias I tampoco se manifiestan variaciones destacables respecto a años anteriores. Las especies más sensibles, como el somormujo lavanco y el aguilucho lagunero, fueron observadas también en la primavera posterior al vaciado aparentemente en las mismas condiciones que durante el prevaciado, aunque no se pudo comprobar la nidificación del primero.

3. En el río Cinca también se aprecia la recuperación, detectándose una diversidad de aves acuáticas similar a la de la primavera del año anterior al vaciado y la presencia de especies que requieren cursos de aguas relativamente limpias, como el zampullín.

\section{Nutria}

Aspectos demográficos

Los elementos más destacables en relación al efecto de los desembalses son los siguientes:

- La comparación de los resultados de las campañas anterior y posteriores al primer desembalse, permitió detectar la ausencia en las prospecciones posteriores, de un macho adulto y de una camada de dos crías que se localizaba en el sector del azud Arias 1. En ninguno de los dos casos 
RIO CINCA

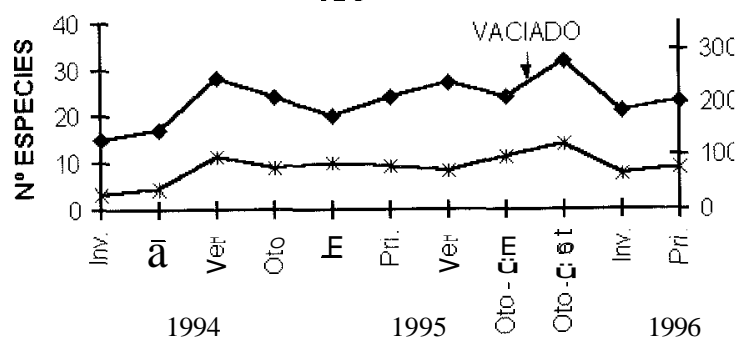

RIO ESERA

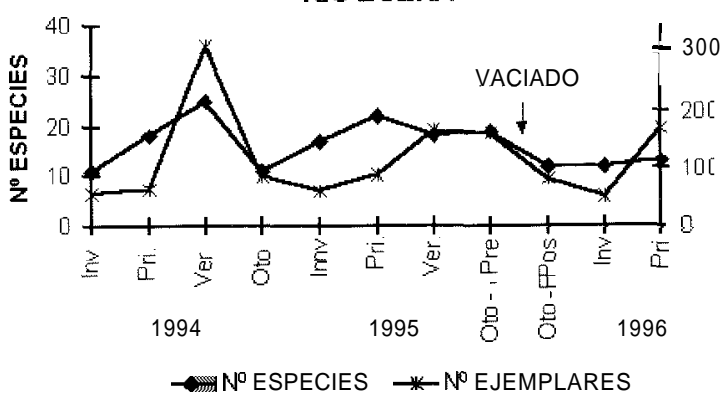

Figura 3. Número de especies de aves y número de ejemplares registrados en las diferentes estaciones fluviales de muestreo. Se separan los censos de otoño de 1995 anterior y posterior al vaciado del embalse. Number of species of birds and number of individuals registered at thr different fluvial survey sites. The censuses of autumn 1995 prior to and following drainage of the reservoir are given separately.

se ha podido establecer con certitud la causa de la desaparición, contemplándose como posibles causas la muerte o la migración, esta última especialmente plausible en el caso del macho adulto.

- Durante la primavera posterior al primer vaciado, se detectan en el Cinca dos nuevas camadas, lo que pone en evidencia la existencia de recursos tróficos suficientes para la reproducción.

- En la primavera posterior al segundo vaciado se constata que la nutria sigue reproduciéndose en la zona, aunque disminuye el número de camadas detectadas respecto a años anteriores ya que sólo se constata la presencia de una cría. Respecto a la base de la población de nutria, (adultos y subadultos), aguas abajo de la presa la situación es similar a la de la primavera de 1996, mientras que en el embalse y en el Esera aguas arriba, se detectan menos individuos.
En general por tanto, aunque se aprecia una disminución en el número mínimo de nutrias que utiliza los tramos afectados después de los desembalses (probablemente debido a la disminución de recursos alimentarios, especialmente acusada en el embalse), se considera que los efectos son reversibles porque diversos individuos adultos y subadultos siguen utilizando los sectores estudiados y además, se considera un indicio positivo el hecho de que la nutria siga reproduciéndose en la zona afectada, después de los dos vaciados.

\section{Uso del hábitat}

La obtención de un Indice Medio de Calidad del Hábitat ( $\mathrm{ICH})$, que refleja las características de cada tramo en relación a los requerimientos ambientales de la nutria (presencia de lugares adecuados para madrigueras y encames, recursos tróficos, etc.), permitió cuantificar estos parámetros y relacionarlos con los indicios de actividad de la especie en cada tramo. Anteriormente al vaciado, se observó que las nutrias utilizaban con más intensidad los tramos con mayor Indice de Calidad del Hábitat, localizándose en ellos una mayor densidad de rastros (Fig. 4).

- En los efectos a corto plazo (un mes después del primer desembalse), se agudiza la relación del uso del hábitat con el Indice de Calidad, observándose:

1. Un incremento de la actividad de nutria en los tramos con mejores condiciones ambientales, destacando especialmente el aumento de actividad observado en el tramo no afectado del río Cinca (entre el Grado y la confluencia) y en los tramos localizados aguas abajo del azud Arias $\mathbf{1 1 .}$

2. En los tramos más directamente afectados por el desembalse, disminuye la actividad de la especie, aunque se sigue detectando la presencia de nutrias en estos sectores (probablemente en desplazamientos exploratorios o de paso hacía otros sectores no alterados), incluso en este período de máxima perturbación. Los tramos que acusan de manera más intensa la disminución de la actividad de nutria son el curso del Esera aguas abajo de la presa y el Cinca, entre la confluencia y el azud Arias 1.

- A medio plazo (seis meses después del primer vaciado):

1. Se detecta una recuperación importante en los Indices de Calidad del Hábitat en la mayor 

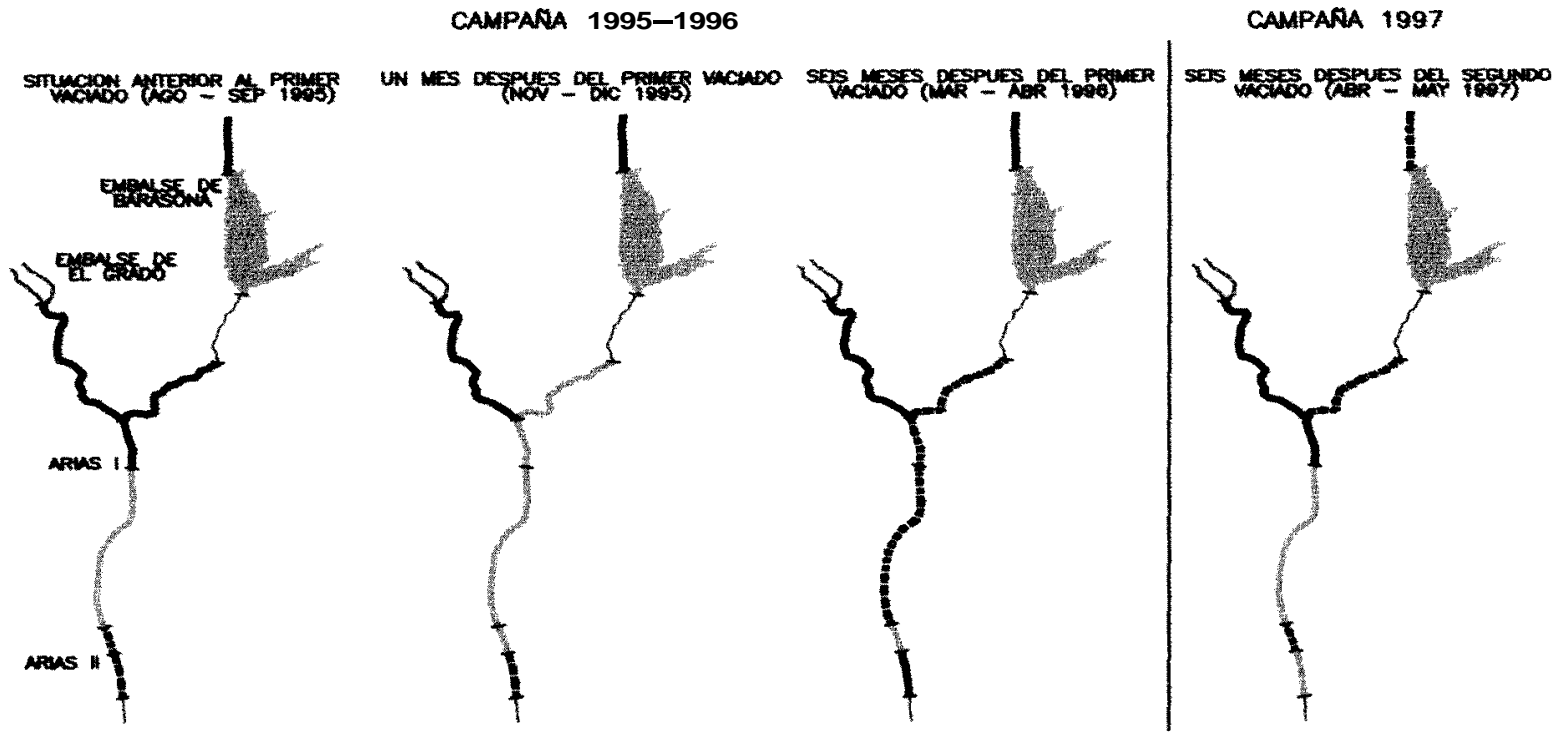

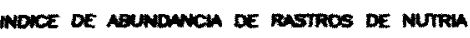

- N.TO (3-4)

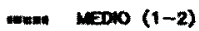

ano (0)

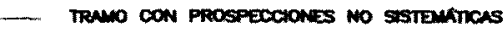

Figura 4. Abundancia de rastros de nutria en los diferentes tramos estudiados. La abundancia se expresa como el número de tipos de rastros que presentan en cada tramo, un valor superior a la media obtenida para ese tipo de rastro en el conjunto de los tramos estudiados. The abundance of otter signs at ihe different siretches studied. The abundance is expressed as the number of types of otter signs which register, in euch sector, a value higher than the average obtained for each type of sign, for all of the stretches surveyed.

parte de los tramos afectados (por efecto del lavado por lluvias torrenciales) y una recuperación paralela de la actividad de la nutria en estas zonas.

2. Destaca Únicamente la baja actividad de nutria en dos sectores: el embalse y el curso del Ésera aguas abajo de la presa. En el primero, este efecto se atribuye a la disminución de peces que constituían su principal recurso trófico, y se desconocen las causas de la baja utilización del Esera, aunque probablemente es debido también a una insuficiente recuperación de los recursos tróficos. También puede considerarse, aunque es poco probable, el efecto de la intensa alteración del tramo final del curso a consecuencia de actividades no directamente relacionadas con el vaciado (construcción de un azud, canal de derivación y obras de acondicionamiento de la carretera paralela a los cauces fluviales).

- La situación en la primavera de 1997 posterior al segundo desembalse, es similar a la obser- vada en la misma estación en 1996 destacándose los siguientes aspectos:

1. Se detecta una concentración de actividad en el tramo del Cinca no afectado por el desembalse, reflejando un uso aún más acusado que en períodos anteriores.

2. Continúa la recuperación de la actividad en el área embalsada Arias I, una zona muy utilizada por la nutria antes de los vaciados, y también se constata un notable aumento de actividad en el río Esera aguas abajo de la presa, principalmente en el tramo próximo a la confluencia.,

3. En el embalse y en el tramo del Esera aguas arriba se detecta en cambio, una disminución notable en la abundancia de los indicadores de uso del hábitat. La baja abundancia de rastros en estos dos sectores se atribuye a la disminución de la biomasa de peces, pero también hay que tener en cuenta el efecto metodológico, ya que la prospección se realizó con el embalse completamente lleno, disminuyendo la superficie adecuada para 

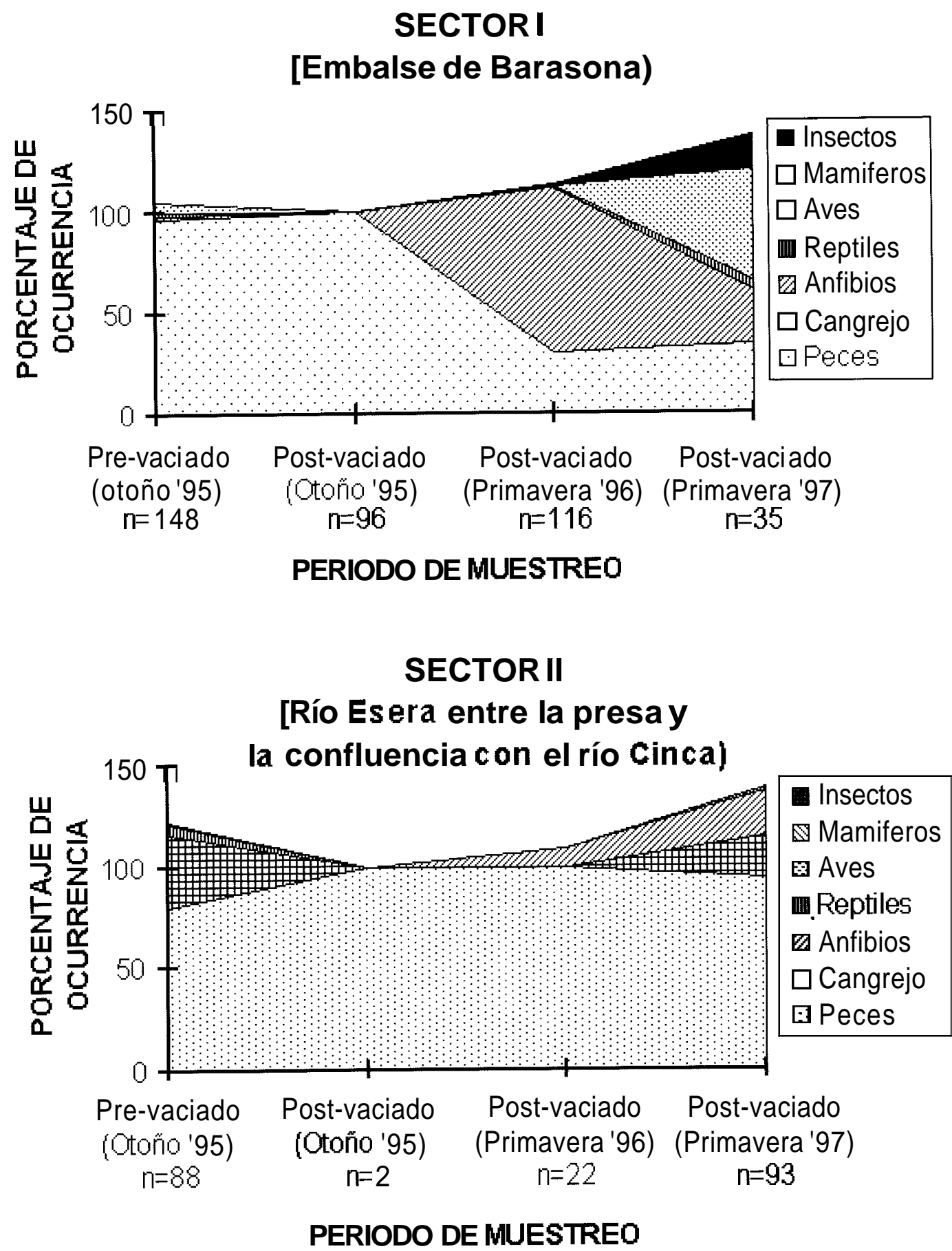

Figura 5. Evolución de la dieta de la nutria en los Sectores I y II durante los cuatro periodos de inuestreo. Changes in otter diet at Sectors I and II during the four survey periods. Data are expressed as percentage occurrences. 
la detección de rastros, respecto a períodos anteriores.

\section{Dieta}

La base de la dieta de la nutria en el río Cinca la constituyen los peces y el cangrejo de río americano (Procambarus clarkii). En total se ha determinado el consumo de diez especies de peces (con dominancia de barbo -Barbus graellsi- y madrilla -Chondrostoma toxostoma-), dos de anfibios (rana común y sapo común), dos de reptiles (culebra de agua -Natrix maura- y serpiente de escalera -Elaphe scalaris-), tres de aves (ánade real, polla de agua -Gallinula chloropus- y lavandera blanca-Motacilla alba-) y una de crustáceos (cangrejo de río). También aparecen en proporciones muy reducidas, micromamíferos, insectos y moluscos. En el embalse y el Ésera aguas arriba de este, no se ha realizado un análisis exhaustivo de las especies consumidas, aunque respecto al Cinca, la principal diferencia es la reducida presencia de cangrejo de río. La evolución de la dieta de la nutria en los distintos sectores estudiados durante los cuatro muestreos se indica en las figuras 5 y 6 .

- A corto plazo, la dieta muestra variaciones destacables:

1. En los tramos fluviales directamente afectados por el vaciado, la diversidad de presas consumidas disminuye considerablemente y la dieta esta constituida casi en exclusiva por peces (las especies más representadas son el barbo, la madrilla y la carpa-Cyprinus carpio-, por este orden). El cangrejo de río prácticamente desaparece de la dieta en estos tramos.

2. En el embalse y en el Ésera aguas arriba de éste, la dieta muestra pocas variaciones con respecto al muestreo previo al vaciado, estando constituida casi totalmente por peces.

3. En el tramo del Cinca anterior a la confluencia, y por tanto no afectado directamente, la dieta es similar a la del período anterior al vaciado.

- A medio plazo, en la primavera y el verano posteriores al vaciado, la dieta evoluciona de manera claramente distinta en los tramos situados aguas arriba y aguas abajo de la presa.

1. Aguas arriba de la presa, el consumo de peces disminuye notablemente, siendo sustituidos por los anfibios, que en el embalse, están presentes en el $81 \%$ de los excrementos localizados.
Estos resultados, no observados en ninguno de los tramos situados aguas abajo del embalse, parecen mantener relación con la baja disponibilidad de peces en este sector, aunque también se relaciona con un incremento de la densidad de anfibios, que en este período acuden a puntos con agua para reproducirse.

2. Aguas abajo de la presa, en los tramos del Ésera y del Cinca después de la confluencia, la diversidad de presas muestra cierta recuperación y el cangrejo vuelve a estar presente. Aún así, la base de la dieta la siguen constituyendo los peces.

- En la primavera 1997, posterior al segundo vaciado, se observa que la dieta es similar a la de la misma estación del 1996, destacando los siguientes aspectos:

1. Aguas arriba de la presa, el consumo de peces sigue sin recuperar los niveles anteriores a los desembalses y en cambio, aumenta el consumo de otras presas alternativas como las aves y los anfibios.

2. Aguas abajo de la presa, destaca en los tramos afectados por los vaciados una notable recuperación de la presencia del cangrejo de río en la dieta acompañada también por una mayor presencia de otras presas como los anfibios. Por otro lado, en la mayoría de los tramos se detecta, con respecto al 1996, una disminución en la presencia de peces en los excrementos, aunque estos siguen siendo en general, la presa principal.

- Globalmente, uno de los resultados más destacables es la disminución de la diversidad de la dieta de la nutria que se aprecia en todos los tramos afectados por el vaciado y la desaparición temporal (posteriormente se recupera) del cangrejo de río americano, que debió resultar fuertemente afectado por el depósito de lodos procedentes del embalse. Otro aspecto relevante, es el importante consumo de peces en los tramos del Cinca situados aguas abajo de la presa, confirmando la presencia de estos en el río incluso en el período de máxima afectación. Cabe destacar en este sentido, que la morfología del cauce y la existencia de canales o acequias paralelas al mismo que no resultaron afectados, han permitido conservar reductos en los que se mantenían hábitats adecuados para los peces y cangrejos. Este efecto ha tenido un interés fundamental porque ha permitido que la nutria siguiera disponiendo de recursos tróficos durante el periodo de afectación del vaciado. Por otra parte, la dieta de la nutria se muestra más alterada 

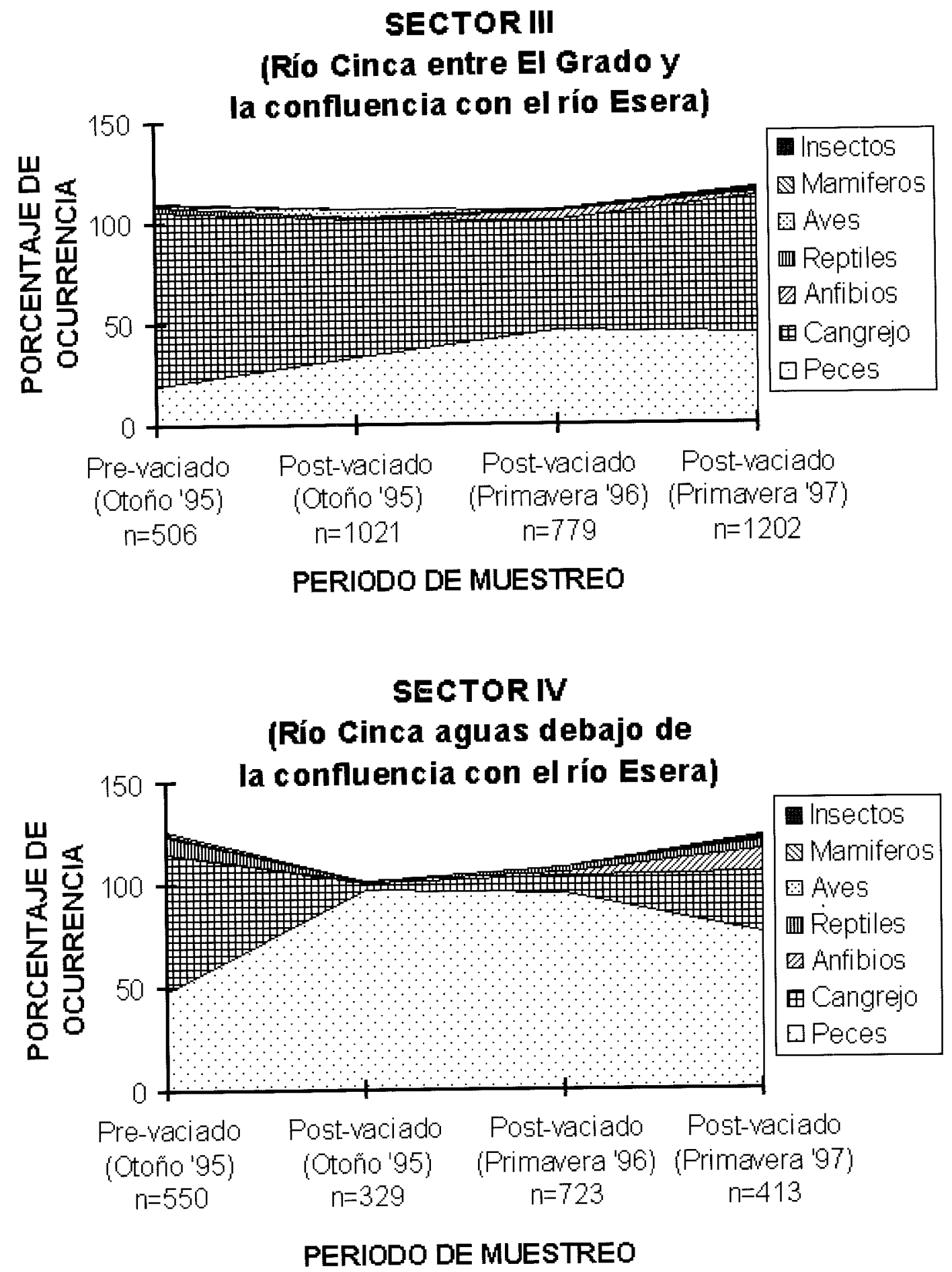

Figura 6. Evolución de la dieta de la nutria en los Sectores III y IV durante los cuatro períodos de muestreo. Changes in otter diet at Sectors III and IV during the four survey periods. Data are expressed as percentage occurrences. 
en el área de estudio aguas arriba de la presa debido a la pérdida de biomasa de peces -su principal recurso trófico-,tanto en el embalse como en los tramo final del Esera.

\section{RECOMENDACIONES SOBRE PREVENCIÓN Y CORRECCIÓN DE IMPACTO EN EL VACIADO DE EMBALSES}

- El diseño de medidas preventivas, correctoras o compensatorias, del vaciado de embalses, debe realizarse de forma particularizada para cada caso, ya que las recomendaciones pueden ser muy diversas en función de:

1. Las características del curso fluvial y su entorno.

2. Las características físicas y químicas de las aguas del embalse y la composición de sus Iodos.

3. Las características de las poblaciones faunísticas afectadas.

- Las operaciones que se recomienda llevar a cabo en cada fase del proyecto son las siguientes:

1. Antes del vaciado es básico elaborar un estudio previo que analice los tres factores indicados y realice la propuesta de las medidas correctoras adecuadas a cada caso.

2. Asimismo, en caso de que el estudio determine la conveniencia de realizar intervenciones previas al vaciado, estas deberán llevarse a cabo con suficiente antelación para permitir que las especies afectadas identifiquen los hábitats acondicionados, los nuevos pasos hacía zonas que no resulten afectadas, etc.

3. Finalmente, durante la fase de vaciado y posteriormente (durante un año como mínimo), deberá aplicarse una monitorización basada en estaciones control y en parámetros indicadores, que permita valorar si los efectos del vaciado se desarrollan en función de lo previsto en el estudio previo o si son necesarias nuevas medidas.

- Entre los factores que más condicionan la magnitud de los efectos del vaciado, se han destacado los siguientes aspectos:

1. Calendario de actuaciones. La época más adecuada para realizar el vaciado de un embalse es el otoño, ya que en ella no se afecta al periodo reproductor de la avifauna acuática ni de los anfibios.

2. Disponibilidad de hábitats alternativos en zonas próximas. Es importante determinar la existencia de estos ambientes y además su capacidad para acoges a los individuos desplazados. En caso que no dispongan de refugios o recursos tróficos suficientes se pueden realizar actuaciones de mejora de hábitats

3. Conservación de sectores no alterados en los tramos directamente afectados. Es fundamental delimitar estrictamente las actuaciones complementarias que se deban realizar (canales, azudes, nuevas captaciones, etc,), evitando al máximo la afectación de formaciones vegetales de ribera y enclaves de interés faunístico estratégico.

4. Minimizar la duración del periodo de máxima afectación. La capacidad de recuperación de las comunidades faunísticas vendrá en gran medida determinada por la duración de la perturbación. La posibilidad de actuar sobre este parámetro será muy distinta según las condiciones particulares de cada embalse; aun así, es importante considerar que cualquier actuación que permita limpiar con rapidez el sedimento acumulado en el lecho fluvial será positiva.

Un importante factor a tener en cuenta en las actuaciones de manejo de hábitats que se implanten con la finalidad de minimizar los efectos del vaciado, es la precaución de analizar previamente -y globalmente- sus consecuencias, para que estas no comporten otros impactos negativos sobre los ecosistemas.

\section{CONCLUSIONES}

La monitorización de los efectos de los vaciados del embalse de Barasona, situado en una zona que contiene elementos faunísticos relevantes (gran diversidad de aves acuáticas y una población de nutria), ha permitido mejorar el conocimiento sobre la respuesta de las comunidades faunísticas a esta perturbación y definir medidas que pueden minimizar el impacto del vaciado de otros embalses. Las principales conclusiones se destacan a continuación.

- El impacto de los vaciados del embalse sobre las comunidades faunísticas estudiadas se ha visto considerablemente atenuado por los siguientes factores:

1. La época en la que se han llevado a cabo, no afectando el período de reproducción de los anfibios y las aves acuáticas. 
2. La existencia de tramos fluviales próximos a los sectores alterados, que conservaron las condiciones previas al vaciado (en este sentido, es remarcable el tramo del Cinca entre el Grado y la confluencia con el Ésera).

3. El mantenimiento de acequias paralelas al Cinca que conservaron buenas condiciones y aportaron aguas limpias al cauce de este curso.

4. La corta duración de la perturbación, ya que las abundantes precipitaciones del invierno de 1995 y la primavera de 1996, realizaron una profunda limpieza de los sedimentos que cubrían el lecho fluvial.

- Los elementos faunísticos que han sido objeto de un seguimiento más detallado (las aves acuáticas y la nutria), mostraron intensos cambios en el uso del espacio en las semanas posteriores al primer vaciado, abandonando la zona de estudio o desplazando su actividad hacía los sectores menos alterados. En las prospecciones realizadas seis meses más tarde, se apreciaba una notable recuperación de la actividad en la mayor parte de zonas afectadas (con la excepción de algunos tramos que muestran un bajo índice de uso por parte de la nutria), y las comunidades faunísticas mostraban una composición similar a la de los muestreos previos al primer vaciado.

- Aunque el vaciado ha comportado la pérdida de un número indeterminado de individuos de las distintas especies, no se han detectado afectaciones irreversibles en las poblaciones de los grupos taxonómicos estudiados. Este efecto se puede explicar en parte, por la adaptación de las especies que habitan en los cursos fluviales a recuperar sus efectivos tras perturbaciones de carácter catastrófico que pueden producirse de manera natural en los ríos, a causa de fuertes avenidas.

- Los efectos en las especies que tienen un baja tasa de renovación y que presentan dificultades de seguimiento poblacional (como es el caso de la nutria), deberían seguir siendo objeto de monitorización, ya que los efectos del vaciado podrían manifestarse a más largo plazo.

\section{BIBLIOGRAFÍA}

BUENO, M. 1993. Resumen de los censos de aves acuáticas invernantes en la provincia de Huesca y áreas limítrofes (1987-1992). Lucas Mallada, 5: 931.
DELIBES, M. 1990. La nutria (Lutra lutra) en Espa$\tilde{n} a$. Ministerio de Agricultura, Pesca y Alimentación. ICONA. Madrid. 198 pp.

DELIBES, M., S.M. MACDONALD, \& C. F. MASON. 1991. Seasonal marking, habitat and organochlorine contamination in otters (Lutra lutra): a comparison between catchment in Andalucia and Wales. Mammalia, 55(4): 567-578.

FALCÓN, J.M. 1982. Los anfibios y reptiles de Aragón Ed. Librería General. Zaragoza.

FALCÓN, J.M. 1987. Nuevas citas de anfibios y reptiles en Aragón. Revista española de Herpetología, 2:83-130.

JOVÉ, S. 1995. La filmación en vídeo infrarojo: una técnica de soporte para el estudio de los mamíferos nocturnos. In: II Jornadas nacionales sobre conservación y estudio de mamíferos, SECEM, 7-10 diciembre 1995. Soria.

LENTON, E.J., P.R.F. CHANIN, \& D.J. JEFFERIES. 1980. Otter survey of England, 1977-79. Nat. Conserv. Council, $75 \mathrm{pp}$.

MACDONALD, S.M. \& C.F. MASON. 1992. Status und conservation needs of the otter (Lutru lutra) in the Western Paleartic. Council of Europe. 66 pp.

MARTÍNEZ RICA, J.P. 1989 El atlas provisional de los anfibios $\mathrm{v}$ reptiles de Españu y Portugal (APA$R E P)$. Presentación y situación actual. Monografías de Herpetología. Asociación Herpetológica Española. Salamanca. 73 pp.

MASON, C.F. \& S.M. MACDONALD. 1986. Otters. Ecology and Consewation. Cambridge Univ. Press. Cambridge.

PEDROCCHI-RENAULT, C. 1978. Las aves de Aragón. Ed. Librería General. Zaragoza.

ROSELL, C., J. PARPAL, R. CAMPENY, S. JOVÉ, A. PASQUINA \& J.M. VELASCO. 1995. Mitigation of barrier effect of linear infrastructures on wildlife. In: Hubitat Fragmentation \& Infrastructure. pp 367-372. 17-21 September 1995, Maastricht-The Hague, The Netherlands.

RUIZ-OLMO, J. 1985. Distribución, requerimientos ecológicos y alimentación de la nutria (Lutra lutra L., 1758) en el $\boldsymbol{N E}$ ibérico. Tesis de licenciatura. Universitat de Barcelona. Inédito.

RUIZ-OLMO, J. 1995. Estudio bionómico de la nutria (Lutru lutra L., 1758) en aguas continentales de la Península Ibérica. Tesis Doctoral, Universidad de Barcelona. Inédito

SEO-ARAGON. 1994. Rocín, anuario ornitológico de Aragón 1991-1992. Ed. Seo-Aragón. Zaragoza. 
SIDOROVICH, V.E. \& G.O. LAUZHEL, 1992.Numbers of otters and approach to population estimation in Byelorussia. IUCN Otter Specialist Group Bulletin. 7: 13-16.

SIDOROVICH, V.E. 1994. About the otter (Lutra lutra) and other mustelids at the Cinca and Ésera river vallevs concerning to the project: "Análisis del impacto del vaciado del embalse de Barasona (Huesca) sobre anfibios, aves y mamíferos vinculados al ambiente de ribera". Dep. Zoología. Univercitat de Barcelona. 